(C) 1982. The Genetical Society of Great Britain

\title{
RECOMBINATION MAINTAINS EQUILIBRIUM FREQUENCIES OF COMMON ALLELES
}

\author{
J. T. MANNING \\ Institute of Extension Studies, University of Liverpool, Liverpool, U.K.
}

Received 7.v.82

\section{INTRODUCTION}

IT is suggested in this note that recombination is necessary to the maintenance of equilibrium frequencies of common alleles. Thus suppose a population is segregating for two common alleles $A$ and $a$. The equilibrium frequency of $A=p$ and $a=q$ and $p>q$. If the population does not show recombination the $a$ chromosomes will tend to become linked to deleterious mutations more rapidly than the $A$ chromosomes (Muller's ratchet). This will tend to decrease the value of $q$ and increase $p$. The process will therefore ensure that equilibrium frequencies cannot be maintained in populations without recombination. The initially most common allele will increase in frequency and the less common allele will be forced to a lower frequency.

\section{RECOMBINATION AND EQUILIBRIUM FREQUENCIES}

It was Muller (1964) who first pointed out that a population without recombination "can never get to contain, in any of its lines, a load of mutation smaller than that existing in its at present least loaded lines". Muller's ratchet was subsequently investigated mathematically by Haigh (1978) and discussed further by Maynard Smith (1978). Consider a haploid population, without recombination, of size $N$. The mutation rate per genome is $U$ and an individual carrying $k$ deleterious mutations has a fitness of $(1-s)^{k}$. The model works only for mildly deleterious mutations so $s$ is assumed to be small. At equilibrium individuals will be present with $0,1,2, \ldots$ mutations. The size of the optimal class i.e., those individuals with 0 mutations is

$$
n_{0}=N e^{-U / s} \text {. }
$$

This is identical for populations with or without recombination. If $n_{0}$ is small it is possible that all individuals in this class may die or fail to reproduce. A population with recombination will regenerate the class but if recombination is absent the class is lost and the new optimal class has one mutation. The size of $n_{0}$ determines the speed at which the ratchet moves in populations without recombination. If it is very small i.e., $n_{0} \leqq 10$, it will move rapidly; if $n_{0} \bumpeq 100$ it will move, although slowly.

Now suppose the population is polymorphic and is segregating for say two alleles $\boldsymbol{A}$ and $a$. The alleles may be maintained by frequency dependent selection. If there is no recombination deleterious mutations will accumulate on the chromosomes carrying $A$ and $a$. However, if the equilibrium frequency of $A=p$ and $a=q$ then it will be exceptional if $p=q$. Suppose therefore that $p>q$, then $n_{0}$ for $A$ chromosomes is

$$
n_{0} A=(N p) e^{-U / s} \text {. }
$$


This is larger than $n_{0}$ for $a$ chromosomes which is

$$
n_{0} a=(N q) e^{-U / s} \text {. }
$$

In populations without recombination the ratchet will move more quickly for $a$ chromosomes than for $A$ chromosomes. As it does, the mean fitness of the former will reduce more rapidly than that of the latter. This will depress $q$ and increase $p$.

\section{REFERENCES}

HAIGH, J. 1978. The accumulation of deleterious genes in a population-Muller's ratchet. Theor. Pop. Biol., 14, 251-267.

MA YNARD SMITH, J. 1978. The Evolution of Sex. Cambridge University, New York.

MULLER, H. J, 1964. The relation of recombination to mutational advance. Mutat. Res., 1, 2-9. 